\title{
Noninvasive mechanical ventilation in patients with myasthenic crisis
}

Original article Seneviratne J et al. (2008) Noninvasive ventilation in myasthenic crisis. Arch Neurol 65: 54-58

\section{SYNOPSIS}

KEYWORDS bilevel positive airway pressure (BIPAP), mechanical ventilation, myasthenia gravis

\section{BACKGROUND}

Noninvasive ventilation might be an alternative to endotracheal intubation in patients with acquired myasthenia gravis who require ventilatory support because of acute respiratory failure (myasthenic crisis).

\section{OBJECTIVES}

To compare the efficacy of conventional endotracheal intubation and mechanical ventilation (ET-MV) with that of noninvasive ventilation with bilevel positive airway pressure (BIPAP) in patients with myasthenic crisis, and to identify predictors of noninvasive ventilation outcome and duration in this setting.

\section{DESIGN AND INTERVENTION}

This was a retrospective review of all patients with myasthenic crisis who were treated at a hospital in the US in the period January 1987 to December 2006. Patients with Lambert-Eaton syndrome or congenital myasthenia and those who had undergone thymectomy were excluded. The final analysis included 60 episodes of myasthenic crisis in 52 patients (mean age 62.6 years, range 17-90 years; $52 \%$ female). All patients had severe generalized and bulbar weakness. Median disease duration from diagnosis to presentation with myasthenic crisis was 4 years (range 1 month to 43 years). The investigators compared outcome between patients who received ET-MV without a preceding trial of BIPAP (36 episodes) and those who were initially treated with BIPAP (24 episodes). Baseline demographic and physiological variables were similar in these two groups. Failure of noninvasive ventilation was defined as a requirement for endotracheal intubation following BIPAP trial.

\section{OUTCOME MEASURES}

The main outcome variable was the duration of mechanical ventilation. The following factors were evaluated as predictors of failure of noninvasive intubation: pulmonary function test results and arterial blood gases on admission and on BIPAP initiation; trigger factors for myasthenic crisis; type of immunotherapy administered; and history of crisis or pre-existing lung disease.

\section{RESULTS}

BIPAP use significantly reduced the duration of ventilation (mean 5.6 days, range 1.5-21.0 days, compared with 13.6 days, range 3-60 days in patients initially treated with ET-MV). Longer ventilation duration was associated with ET-MV use $(P=0.02)$, lower maximal expiratory pressure on arrival $(P=0.02)$ and the development of atelectasis $(P<0.005)$. Initial BIPAP treatment emerged as the only variable associated with decreased ventilation duration $(P<0.007)$. Noninvasive ventilation also reduced the lengths of stay in the intensive care unit (ICU) and hospital by a third each (median 7 days; $P<0.001$ for both). In 14 (58\%) cases initially treated with BIPAP, endotracheal intubation was avoided. The mean duration of ventilation in these cases was $4.3 \pm 2.9$ days. Patients in whom BIPAP was successful had significantly lower rates of pulmonary complications (atelectasis and pneunomia) than did patients who underwent endotracheal intubation $(21 \%$ vs $54 \% ; P=0.04)$. The only factor associated with BIPAP failure was a $\mathrm{pCO}_{2}$ level $>45 \mathrm{mmHg}$ on BIPAP initiation $(P=0.04)$.

\section{CONCLUSION}

BIPAP is effective for the treatment of myasthenic crisis and can prevent intubation and prolonged ventilation, thereby reducing the risk of pulmonary complications and the lengths of ICU and hospital stay. 


\section{GOMMENTARY}

\section{Anthony A Amato}

A recent review of 1,976 patients with myasthenia gravis reported that $19 \%$ of patients developed myasthenic crisis (severe bulbar weakness or ventilatory failure). ${ }^{1}$ The treatment of myasthenic crisis is twofold. First and foremost is maintenance of the airway and provision of ventilatory support. Second is treatment of the underlying autoimmune disease with plasma exchange or intravenous immunoglobulin, coupled with immunosuppressive therapy. Before the availability of antibiotics to treat pneumonia and the discovery of anticholinesterase medications, 70\% of patients with myasthenia gravis died from pneumonia or respiratory failure (hence the term 'gravis'). ${ }^{1}$ With better ICUs and the availability of mechanical ventilation, mortality from myasthenia gravis fell from $30 \%$ in the 1950 s to $15 \%$ by the mid-1960s. ${ }^{1}$ The introduction from the late 1960s of corticosteroids, plasma exchange, and other forms of immunotherapy has resulted in a decrease of mortality to $6 \% .^{1}$

Noninvasive ventilation is a more recent breakthrough in the management of patients with respiratory muscle weakness. One mode of therapy increasingly employed in a variety of neuromuscular diseases (e.g. amyotrophic lateral sclerosis and muscular dystrophy) is BIPAP. There have been a few small reports of BIPAP used to treat acute ventilatory failure in myasthenia gravis in the hope that intubation could be avoided or to assist in weaning the patient from the ventilator. ${ }^{2-4}$

The aim of the present study was to compare the effectiveness of ET-MV with that of treatment with BIPAP, and to identify features that may predict success or failure of BIPAP. The authors retrospectively reviewed 60 episodes of myasthenic crisis requiring mechanical ventilation in 52 patients. The initial mode of ventilatory support was BIPAP in 24 episodes and ET-MV in 36 episodes. Of the patients who were initially treated with BIPAP, 10 had to eventually be intubated but 14 avoided intubation. The only predictor of BIPAP failure was a $\mathrm{pCO}_{2}$ level exceeding $45 \mathrm{mmHg}$ on BIPAP initiation.
Patients treated with BIPAP had shorter stays in the ICU (7 days vs 17 days) and in hospital (13 days vs 20 days) than those treated initially with intubation.

The study is limited by its retrospective nature. The decision to intubate or treat with BIPAP was at the discretion of the treating physician. There did not seem to be significant clinical differences at baseline between patients who were intubated and those who were treated with BIPAP. However, care must be taken in drawing the conclusion that those treated with BIPAP had shorter hospital stays just because they were not intubated. It is possible that the patients who were judged to need intubation had or subsequently developed more-severe ventilatory weakness than did patients who were judged not to need intubation, which could explain the prolonged hospital stays. In addition, patients who were intubated might have had more-severe bulbar difficulties (e.g. severe dysphagia). Finally, the number of patients with concomitant lung disease, and the number who had an infection as the trigger for myasthenic crisis, were each twice as high among those in whom BIPAP failed than among those in whom this procedure was successful.

Nevertheless, this study does support the notion that some patients with myasthenia gravis in crisis or impending crisis might be managed with BIPAP. These patients still need to be monitored very closely, particularly if they have underlying lung disease or exacerbation related to infection.

\section{References}

1 Grob D et al. (2008) Lifetime course of myasthenia gravis. Muscle Nerve 37: 141-149

2 Mazia CG et al. (2003) BiPAP in acute respiratory failure due to myasthenic crisis may prevent intubation. Neurology 61: 144

3 Rabinstein AA and Wijdicks EF (2003) Weaning from the ventilator using BiPAP in myasthenia gravis. Muscle Nerve 27: 252-253

4 Rabinstein A and Wijdicks EF (2002) BiPAP in acute respiratory failure due to myasthenic crisis may prevent intubation. Neurology 59: 1647-1649

AA Amato is the Vice-Chairman for Clinical Affairs and Chief of Neuromuscular Division at Brigham and Women's Hospital and Professor of Neurology at Harvard Medical School, Boston, MA, USA.
Acknowledgments

The synopsis was written by Martina Habeck, Associate Editor,

Nature Clinical Practice.

\section{Competing interests}

The author declared no competing interests.

\section{Correspondence}

Brigham and Women's Hospital

Department of Neurology

75 Francis St

Boston

MA 02115

USA

aamato@partners.org

Received 11 February 2008 Accepted 5 March 2008 Published online 22 April 2008

www.nature.com/clinicalpractice doi:10.1038/ncpneuro0793
PRACTICE POINT Myasthenic crisis with ventilatory failure may be managed in some patients with a trial of bilevel positive airway pressure, which could reduce the need for intubation 